\title{
Rehabilitation of an athelete with Kienbock's disease
}

\author{
Janis Yeo Siew Ting \\ From 10th Congress of the Asia-Pacific Federation of Societies of Surgery fo the Hand and the 6th Congress \\ of Asia-Pacific Federation of Societies of Hand Therapists \\ Kuala Lumpur, Malaysia. 2-4 October 2014
}

\section{Introduction}

Kienböck's disease, a vascular necrosis of the lunate, has remained controversial in its basic etiology, natural history and therefore treatment.

\section{Purpose}

This case report describes the hand therapy process of improving the range and strength of a 17-year-old female gymnast to return her to competitive gymnastic training and the competition arena.

\section{Methods}

17-year-old female gymnast, left hand dominant, started hand therapy 8 weeks after a vascularised medial femoral trochlea osteochrondral flap reconstruction to her right wrist.

Initial status, 8 weeks post-operation: AROM fingers full; AROM wrist extension $20^{\circ}$; AROM wrist flexion $15^{\circ}$; AROM wrist radial deviation $8^{\circ}$; AROM wrist ulnar deviation $8^{\circ}$; AROM pronation $70^{\circ}$; AROM supination $90^{\circ}$; QuickDASH disability / symptom score 47.7; QuickDASH sports module score 100. Patient underwent bi-weekly therapy sessions for first 4 weeks focusing on dartthrowers motion, A/PROM, therapeutic exercise and modalities. This is followed by weekly sessions focusing on strengthening and simulation of gymnastic training.

\section{Results}

16 weeks post-operation: AROM fingers full; AROM / PROM wrist extension $45^{\circ} / 50^{\circ}$; AROM / PROM wrist flexion $35^{\circ} / 50^{\circ}$; AROM wrist radial deviation $15^{\circ}$; AROM wrist ulnar deviation $25^{\circ}$; AROM pronation $80^{\circ}$; AROM supination $90^{\circ}$; right grip / left grip strength $13.3 \mathrm{~kg} /$ 21.7kg; QuickDASH disability / symptom score 6.8; QuickDASH sports module score 50. \section{Singapore

Department of Occupational Therapy, Tan Tock Seng Hospital, 308433, 